\title{
ARTICLE \\ Design of Automatic Cutting and Coating Collection System Based on Optical Fiber
}

\author{
Shengchang Lin* Wujiu Bei Jirong Sun \\ Nantong Institute of Technology, Nantong, Jiangsu, 226002, China
}

\section{ARTICLE INFO}

Article history:

Received : 8 August 2018

Revised : 10 September 2018

Accepted : 9 October 2018

Published Online : 16 October 2018

\begin{abstract}
In order to meet the requirement of fiber splicing, the problem of low efficiency and manual operation is adopted. The device is designed and fabricated to meet the convenience and convenience of splicing and equipping in optical fiber production. The simulation design and control system design of SolidEdge are discussed. At present, there is no such device in the market, and it has certain value in optical fiber equipment.
\end{abstract}

Keywords:

Splicing device

PLC FX3u-32 Mr

Telescopic cylinder

Remote monitoring

\section{Introduction}

$\mathrm{O}$ ptical fiber in the transmission of information has a large capacity per unit time, it can load more information, the speed and quality in the process of using optical fiber communication is far higher than that of traditional cable and optical fiber cable, high effectiveness and particularity, in order to make optical fiber production equipment to the greater degree of automation, can improve the shear rate of the fiber.

Therefore, the research, development and production of automatic optical fiber shearing and coating collection system runs accurately, reliably, stably, intelligently, and has reproducibility.

(1) Apply mechanical design and manufacturing and au- tomation technology to design an automatic collection system for optical fiber cutting and coating overflow.

(2) The design of this system will take into account the space possibility of production line body, and optimize the design from the aspects of reliability, stability, security, low noise and intelligence.

(3) The system designed shall be installed on the existing productive equipment, so the structural design, electrical control system and appearance coordination of the device shall fully consider the matching with the existing production line body, without affecting the normal operation of the production equipment, and shall be integrated with it. This system will form the tooling standard, with replicable technical characteristics.

*Corresponding Author:

Shengchang Lin,

Nantong Institute of Technology,

No. 14 Yongxing Road, Gangzha Economic Development Zone, Nantong, Jiangsu, 226002, China.

E-mail: 3457940140@qq.com. 


\section{Research Objectives}

Using PLC, sensor, solenoid valve, pneumatic components and man-machine interface, the device includes: optical fiber shear interceptor system, paint overflow collection subsystem. In the optical fiber shearing and truncation system, when the production of the optical fiber in this unit is finished, the instruction is issued to cut the optical fiber automatically. When the optical fiber shear is finished, the scissor system will reset automatically. In the coating overflow collection subsystem, when the optical fiber is automatically cut off, the paint collection system will automatically extend out the container and collect the overflow coating to prevent the coating from flowing into the curing furnace. The task is automatically reset after completion.

\section{Product Digital Design}

Determine the installation size of the equipment, draw the drawings, and then divide the equipment into base, manipulator, middle reservoir, cutting parts, cylinder and air hole according to the drawings and specific models.

\subsection{The SolidEdge Simulation}

The base is a simple part drawing that can be made by stretching. First draw a 2-dimensional rectangle and then stretch $1 \mathrm{~mm}$ to draw a $2-\mathrm{d}$ triangle that satisfies the drawing on the wide side of the rectangle and extend $1 \mathrm{~mm}$ inward to draw the same pattern on the other side using symmetry. Select one of the triangles as the current surface; draw the drawing below the rectangle and stretch. The simulation diagram is shown in Figure 1.

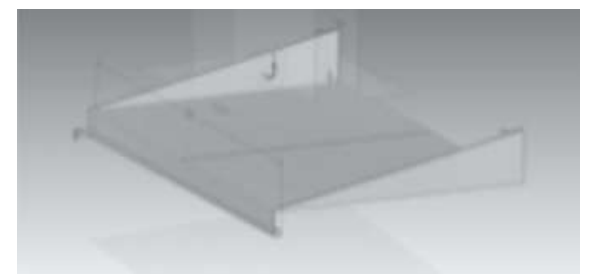

Figure 1. Base Diagram

The manipulator is assembled from five different parts. Claw part, fuselage part, connecting parts: draw three connecting parts directly. The simulation figure is shown in Figure 2.

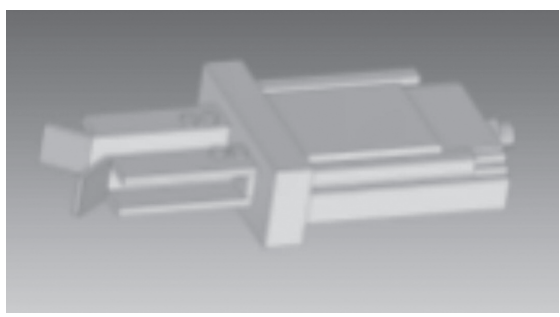

Figure 2. Manipulator Diagram
The middle tank and the desired scissors and their cylinders can be subdivided into four parts: Storage tank, cylinder, scissors, and parts for assembly.

Liquid storage tank: the liquid storage tank is composed of a rectangular tank in the outer shell and the inner one, which is connected with the cylinder. When drawing the shell of the liquid storage tank, draw a two-dimensional pattern of the front image and stretch it. The three are assembled to get the reservoir. The simulation diagram is shown in Figure 3.

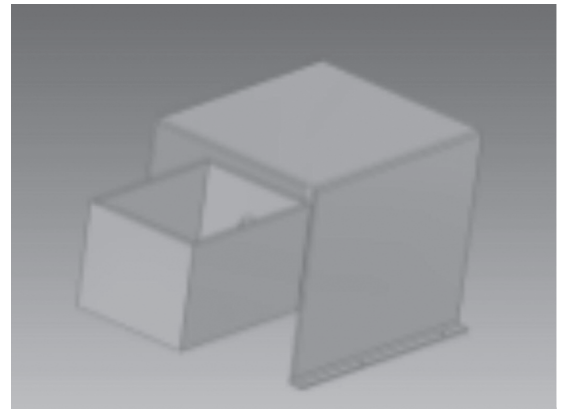

Figure 3. Reservoir Diagram

In the drawing of the cylinder, the rectangle is first drawn to form the rounded Angle at the 4 corners and then the required $3 \mathrm{~d}$ drawing is obtained by drawing and drawing. The simulation diagram is shown in Figure 4.

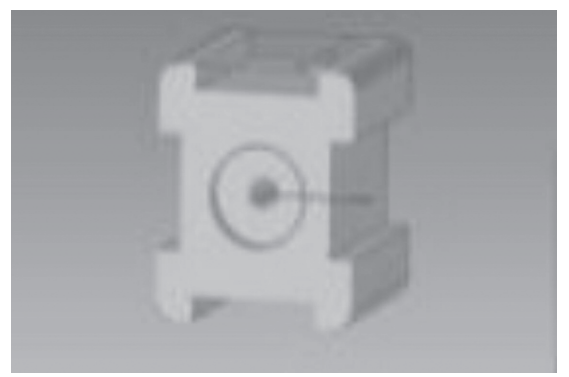

Figure 4. Cylinder Diagram

The scissors stretch in two dimensions and draw movable joints at key points. The opening and closing of scissors is realized through the expansion of the cylinder, and the simulation figure is shown in Figure $5 .^{[1]}$

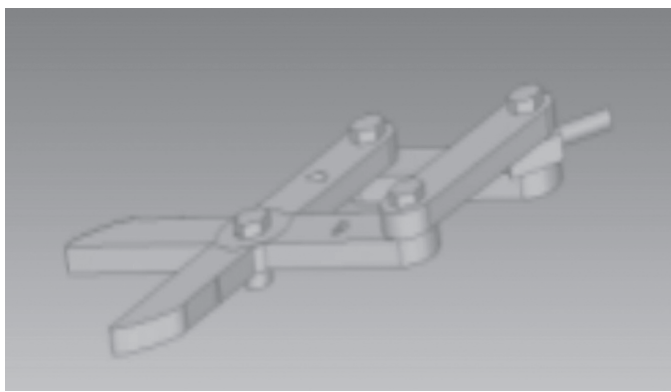

Figure 5. Scissor Diagram 
The first clamping mechanism, which can be divided into 5 small parts, respectively make $2 \mathrm{~d}$ drawing and drawing material removal of each drawing. The simulation diagram is shown in Figure 6.

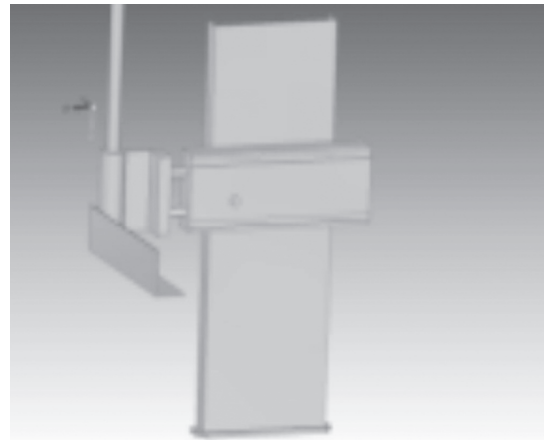

Figure 6. First Clamping Mechanism Diagram

The second clamping mechanism, this part can be composed of two parts to form the manipulator part and the connecting part of the connecting part by the cylinder and several bolts. The simulation diagram is shown in Figure 7.

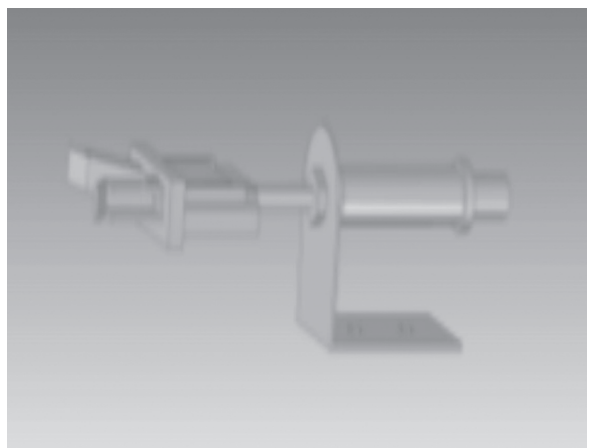

Figure 7. Second Clamp Mechanism Diagram

Stomata, this part is made of several simple parts spliced together. The simulation diagram is shown in Figure 8.

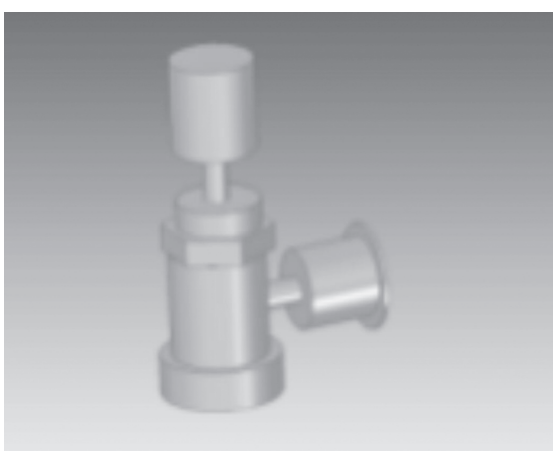

Figure 8. Stomatal Diagram

\subsection{SolidEdge Simulation Diagram}

The product adopts solidedge to realize the simulation, which consists of five parts. The simulation physical diagram of the product is shown in Figure 9.

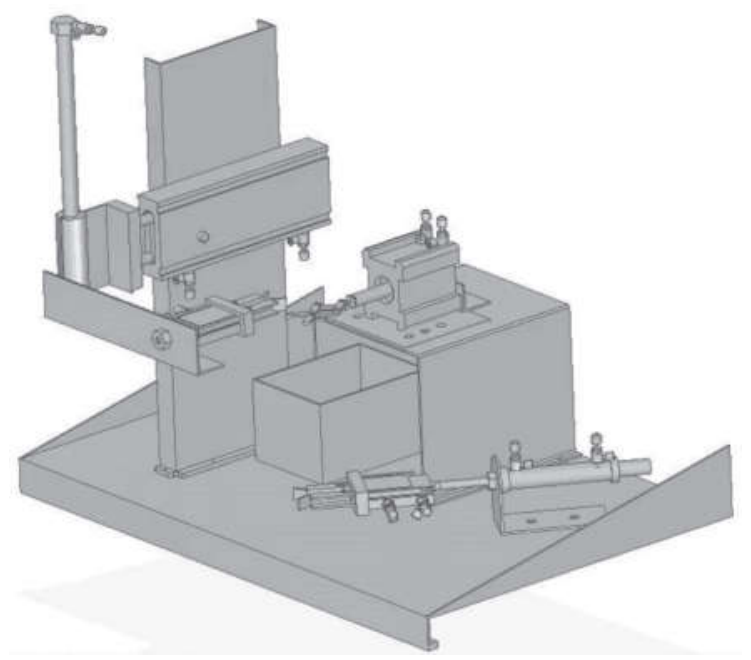

Figure 9. Simulates a Physical Image

\section{System Hardware Design}

According to the required fiber drawing shears with fiber coating, which are used for cutting fiber, the fiber drawing shears with fiber coating include:

(1) Installation: the first clamping mechanism and the second clamping mechanism are set up. The first clamping mechanism and the second clamping mechanism are arranged at intervals on the mounting pedestal. The cutting mechanism is located on the mounting pedestal, and the cutting mechanism is located between the first clamping mechanism and the second clamping mechanism to shear when the first clamping mechanism and the second clamping mechanism clamp the fiber to be snipped respectively.

(2) Base: the base is set on the mounting seat and connected with the mounting seat.

(3) Cutting parts: the cutting parts are set on the base and connected to the base. The third driver is connected to the cutter to drive the cutter to cut the fiber.

The base is limited to a receiving cavity, and the base also includes a receiving box. The receiving box is located in the receiving cavity and is movable in the receiving cavity.

\subsection{Hardware Block Diagram}

When the instructions of the whole device are initiated and relevant operators click the relevant operation button on the touch screen, the touch screen is connected to the PLC through the signal line, and the input instructions of the operator are sent to the PLC indirectly. In this case, the PLC will perform the corresponding input in sequence, thus driving the movement of the whole system. ${ }^{[2]}$ the overall PLC frame diagram is shown in Figure 10. 


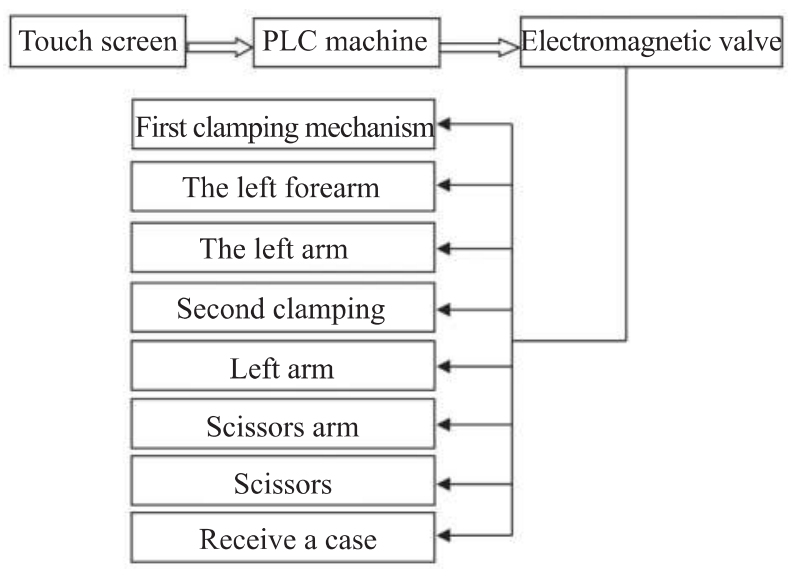

Figure 10. Hardware block diagram

\section{System Software Design}

The system is required to adopt PLC control technology, accurate and reliable, and can modify the program according to the requirement of intelligent production and control.

\subsection{Control Strategy}

The power component is connected with the first clamping mechanism, the second clamping mechanism and the cutting mechanism respectively to be powered by the power component $^{[3]}$. The connected solenoid valve set, relay and switch, solenoid valve set, relay and switch are respectively connected with the stabilized voltage source. According to the control requirements, the program flow chart of the programmable controller is shown in Figure 11.

According to the requirements of the implementation example, the fiber drawing shears with fiber coating can effectively cut the fiber to be sheared through the cooperation of the first holding mechanism, the second holding mechanism and the cutting mechanism. The fiber drawing shears with fiber coating can have simple structure, intelligent control and high cutting efficiency.

\subsection{Program Design}

The control system of the device is implemented by mitsubishi PLC and its I/O distribution table is shown in Table 1 .

\subsection{Features}

Test the different fibers on our equipment and modify our structure to meet the specified distance of the shear fibers. By setting four buttons to operate, the device can be suspended in different working states to test the stability and reliability of the device. When you want to resume after a pause, you can click run and continue working in the same state. You can click the stop button to see if the device stops working after a given week of operation.

\subsection{Test Scheme}

According to the operation requirements of the optical fiber production equipment, how to realize the automatic cutting of the optical fiber is studied, and the coating overflow used for coating the optical fiber will be collected automatically according to the production process setting requirements, laying a foundation for the final realization of the intelligent production line. The "optical fiber automatic cutting and coating collection system" developed by the project is accurate, reliable, stable, intelligent and reproducible. We can test different optical fibers on our equipment and modify our structure to meet the specified distance of the shear fiber. Then, the four buttons we set

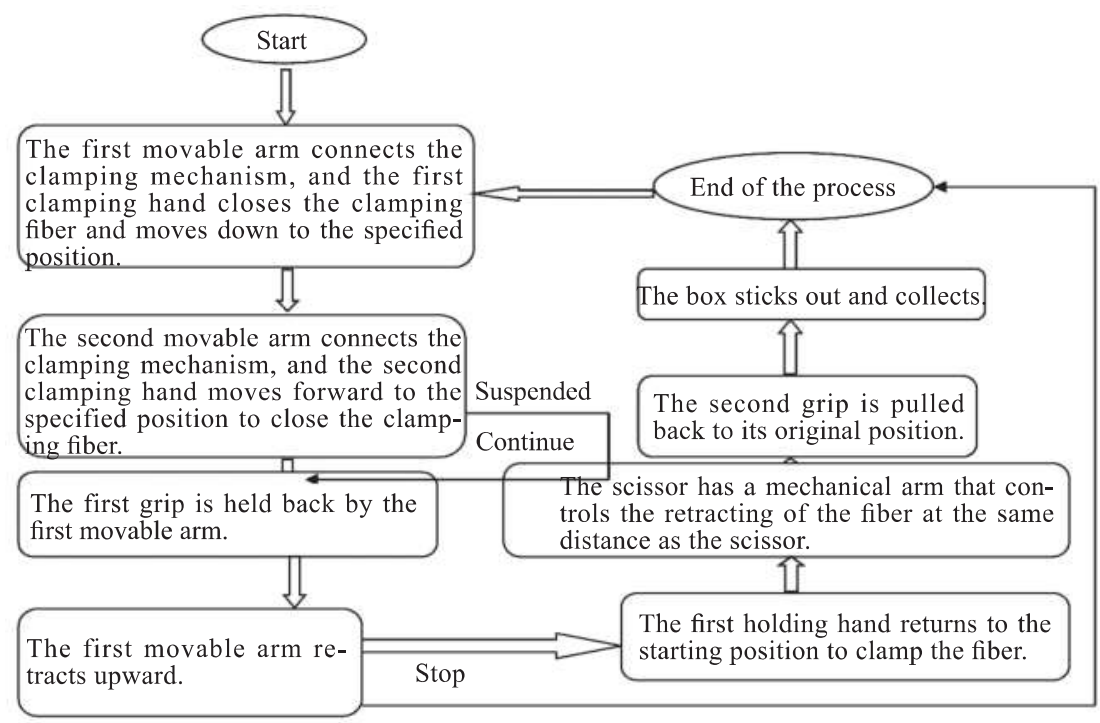

Figure 11. Program Flow Diagram 
Modern Electronic Technology Volume 2 Issue 3 • October 2018

Table 1. I/O Allocation Table

\begin{tabular}{|c|c|c|c|c|c|}
\hline \multicolumn{3}{|c|}{ Input signal } & \multicolumn{3}{|c|}{ output signal } \\
\hline The name of the & Code name & Output point number & The name of the & Code name & Output point number \\
\hline Start the button & SB1 & X001（M1） & $\begin{array}{c}\text { The manipulator arm extends } \\
\text { downwards and the first clamping } \\
\text { hand catches the fiber }\end{array}$ & YV1 & Y001 \\
\hline Stop the button & SB2 & $\mathrm{X} 002(\mathrm{M} 2)$ & $\begin{array}{l}\text { the second gripper extends hori- } \\
\text { zontally forward to hold the fiber }\end{array}$ & YV2 & Y002 \\
\hline Pause the button & SB3 & X003 ( M3) & $\begin{array}{l}\text { The first gripper loosens the optical } \\
\text { fiber mechanical arm and stretches } \\
\text { it out }\end{array}$ & YV3 & Y003 \\
\hline \multirow[t]{5}{*}{ Continue the button } & SB4 & X004 (M4) & The arm retracts upward & YV4 & Y004 \\
\hline & & & $\begin{array}{c}\text { The robot arm retracts inward from } \\
\text { the first clamping hand holding the } \\
\text { fiber }\end{array}$ & YV5 & Y005 \\
\hline & & & Scissors stick out to cut the fiber & YV6 & Y006 \\
\hline & & & $\begin{array}{c}\text { The second grip retracts to its } \\
\text { original position }\end{array}$ & YV7 & Y007 \\
\hline & & & Box out & YV8 & Y010 \\
\hline
\end{tabular}

can be used for operation, and the device can be suspended in different working states to test the stability and reliability of the device. When suspended, you can click run to restore and continue working in the same state.

\section{Conclusion}

In view of the investigation of the fiber industry, manpower is the main factor in the fiber cutting, and the whole process is handled by human. From cutting to recycling drops, this is inefficient and inaccurate. The advent of our devices has brought these processes together, greatly reducing the amount of time it takes to cut, and increasing efficiency many times over. When our products are put into the market, it will change the entire fiber cutting industry, which is of considerable interest, and the market share is unimaginable.

\section{References}

[1] Rong zhao, Weidong Guo, Wenyun Zhang. SolidEdge ST10[M]. Beijing: machinery industry press, 2018, 01. (in Chinese)

[2] Shengyu Zhang. Practical training program for programmable controllers[M]. Beijing: electronic industry press, 2012, 03. (in Chinese)

[3] Qinghong Wu. Practical operating instructions for inverter, programmable controller and touch screen integrated application technology[M]. Beijing: mechanical engineering press, 2015, 05. (in Chinese) 